\title{
ASYMPTOTIC STABILITY OF VISCOUS SHOCK WAVE FOR A ONE-DIMENSIONAL ISENTROPIC MODEL OF VISCOUS GAS WITH DENSITY DEPENDENT VISCOSITY*
}

\author{
AKITAKA MATSUMURA $^{\dagger}$ AND YANG WANG $^{\ddagger}$
}

\begin{abstract}
In this paper we investigate the asymptotic stability of viscous shock wave for a onedimensional isentropic model of viscous gas with density dependent viscosity by a weighted energy method developed in the papers of Matsumura-Mei (1997) and Hashimoto-Matsumura (2007). Under the condition that the viscosity coefficient is given as a function of the absolute temperature which is determined by the Chapman-Enskog expansion theory in rarefied gas dynamics, any viscous shock wave is shown to be asymptotically stable for small initial perturbations with integral zero. This generalizes the previous result of Matsumua-Nishihara (1985) where the viscosity coefficient is given by a constant and a restriction on the strength of the viscous shock wave is assumed. This also analytically assures the spectral stability in the Zumbrun's theory for any viscous shock wave in our specific case.
\end{abstract}

Key words. Asymptotic stablility, viscous shock wave, viscous gas.

AMS subject classifications. 35Q30, 76N10, 35B40.

1. Introduction. In this paper, we investigate the asymptotic stability of viscous shock wave for a one-dimensional isentropic model of viscous gas which has the form in the Lagrangian mass coordinates

$$
\left\{\begin{array}{l}
v_{t}-u_{x}=0, \\
u_{t}+p(v)_{x}=\left(\mu(v) \frac{u_{x}}{v}\right)_{x}, \quad t \geq 0, x \in R, \\
p(v)=\frac{a}{v^{\gamma}}
\end{array}\right.
$$

where $v(>0)$ is the specific volume, $u$ is the fluid velocity, $p=p(v)$ is the pressure, $\gamma(\geq 1)$ is the adiabatic constant, $a$ is a positive constant, and $\mu=\mu(v)$ is the viscosity coefficient which is assumed to be a positive smooth function of $v$.

It is easily seen that the system (1.1) admits a traveling wave solution with shock profile (in what follows we call "viscous shock wave")

$$
(v, u)=(V, U)(x-s t), \quad(V, U)( \pm \infty)=\left(v_{ \pm}, u_{ \pm}\right)
$$

under some physical conditions (Rankine-Hugoniot and entropy conditions), where $s$ is the shock speed and $v_{ \pm}>0, u_{ \pm} \in R$ are the given far field states. We are interested in the asymptotic stability of the viscous shock wave, which has been very important problem from both mathematical and physical points of view. We first summarize the developments on this problem in the case $\mu$ is a positive constant. It is noted that as long as small amplitude viscous shock wave is concerned, this case is essentially same as the density dependent one. First of all, Matsumura-Nishihara [8](1985) showed that there exist a positive constant $C\left(v_{-}, \gamma\right)$ with $C \rightarrow \infty$ as $\gamma \rightarrow 1$ such that if

\footnotetext{
${ }^{*}$ Received May 22, 2010; accepted for publication October 12, 2010.

$\dagger$ Department of Pure and Applied Mathematics, Graduate School of Information Science and Technology, Osaka University, Toyonaka, Osaka 560-0043, Japan (akitaka@math.sci.osaka-u.ac.jp). Matsumura's research is supported in part by Grant-in-Aid for Scientific Research (B) 19340037, Japan.

‡Software Division, Hitachi Ltd., Yokohama, Kanagawa 244-8555, Japan (caeserwangcn@hotmail .com).
} 
$\left|v_{+}-v_{-}\right| \leq C\left(v_{-}, \gamma\right)$, then the viscous shock wave $(V, U)(x-s t)$ is asymptotically stable for small initial perturbations with integral zero. This reads that for $\gamma=1$ (isothermal case), any viscous shock wave is asymptotically stable, but for $\gamma>1$, a restriction on the amplitude of the viscous shock wave is imposed for the asymptotic stability. Later on, there appeared many works treating initial perturbations with nonzero integral (and also general systems of viscous conservation laws). In particular, the asymptotic stability for small amplitude viscous shock wave of (1.1) and related physical systems has been treated in Mascia-Zumbrun [7](2004), and a similar result has been announced by Liu-Zeng [5](2009) in their treatment of systems with artificial viscosity. It also should be noted that the theory Zumbrun and his collaborators developed shows that for our system (1.1) the spectral stability of the linearized system implies the non-linear asymptotic stability for general initial perturbations, and the asymptotic stability for the restricted initial perturbations with integral zero implies the spectral stability. As for the asymptotic stability for large amplitude viscous shock wave, it had been a long standing open problem, except the $\gamma=1$ case in [8]. Recently, Barker-Humpherys-Laffite-Rudd-Zumbrun [1] and HumpherysLaffite-Zumbrun [4] analytically established the spectral stability of sufficiently large amplitude viscous shock wave for the properly rescaled system of (1.1) with physically reasonable adiabatic constant, and also carried out numerical studies indicating the asymptotic stability for intermediate amplitude as well. However, except for the small and large amplitude limits, it still remains open whether viscous shock wave can be analytically shown to be asymptoticaly stable.

In this paper, we investigate the case the viscosity coefficient is depending on the density $\rho=1 / v$ under a physically natural background. According to the Chapman-Enskog expansion theory in rarefied gas dynamics (cf. Chapman-Cowling [2], Kawashima-Matsumura-Nishida [6]), the viscosity coefficient is given by a function of the absolute temperature $\theta$, depending on the assumptions on molecular interaction. The typical two examples are given as follows:

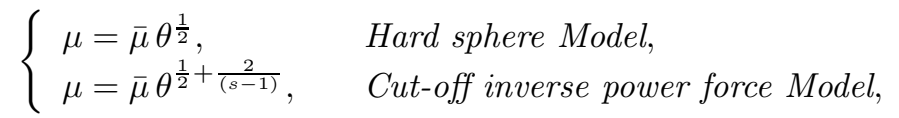

where $s(\geq 5)$ and $\bar{\mu}(>0)$ are some constants. It is noted that the limit as $s \rightarrow \infty$ of the inverse power force model coincides with the hard sphere model, and the case of the minimum $s=5$ is called "Maxwell gas" model. Then, since $\frac{1}{2}+\frac{2}{(s-1)}>\frac{1}{2}$, the above two models are unified as

$$
\mu=\bar{\mu} \theta^{\beta} \quad\left(\beta \geq \frac{1}{2}\right) .
$$

On the other hand, since our model is isentropic, it holds

$$
p=R \rho \theta=R \frac{\theta}{v}=a v^{-\gamma}, \quad(R: \text { gas constant })
$$

which implies

$$
\theta=\frac{a}{R} v^{-(\gamma-1)} .
$$

Hence it follows from (1.4) and (1.6) that

$$
\mu=\mu_{0} v^{-(\gamma-1) \beta} \quad\left(\beta \geq \frac{1}{2}, \mu_{0}=\bar{\mu}\left(\frac{a}{R}\right)^{\beta}\right) .
$$


Now set $\alpha=(\gamma-1) \beta$, then it should be emphasized that the condition $\beta \geq \frac{1}{2}$ is equivalent to $\alpha \geq \frac{1}{2}(\gamma-1)$. Thus, in what follows, we assume that the viscosity coefficient $\mu(v)$ has the form

$$
\mu=\mu(v)=\mu_{0} v^{-\alpha} \quad\left(\alpha \geq \frac{1}{2}(\gamma-1), \mu_{0}>0: \text { constants }\right) .
$$

In this paper, we shall show that under the condition (1.8) any viscous shock wave is asymptotically stable for small initial perturbations with integral zero. It should be noted that this result analytically assures the spectral stability in the Zumbrun's theory for arbitrary viscous shock wave of our system (1.1) under the condition (1.8). The proof is given by a weighted energy method developed in the papers of MatsumuraMei [9] and Hashimoto-Matsumura [3]. In the section 2, we precisely state our main theorem after recalling the arguments on the existence of the viscous shock wave. For the proof, in the section 3, we reformulate the problem in terms of the anti-derivatives of the deviation functions from the viscous shock wave. We establish the a priori estimates by a weighted energy method in the section 4 . Finally in the section 5 , we give a remark that our arguments can be extended to models with more general viscosity coefficient like the Sutherland's model.

Some Notations. $L^{2}$ denotes the Lebesgue space of square integrable functions on $R$, with the standard norm $\|\cdot\| \cdot H^{k}(k=0,1,2, \ldots)$ denote the usual Sobolev's spaces on $R$, with the standard norm $\|\cdot\|_{k}$. Note that $L^{2}=H^{0}$ and $\|\cdot\|=\|\cdot\|_{0}$.

2. Main Theorem. In this section, we formulate the Cauchy problem under the assumption of (1.8), and state our main theorem. Plugging the assumption (1.8) into (1.1), we rewrite the system (1.1) as

$$
\left\{\begin{array}{l}
v_{t}-u_{x}=0, \\
u_{t}+p(v)_{x}=\mu_{0}\left(\frac{u_{x}}{v^{\alpha+1}}\right)_{x}, \quad t \geq 0, x \in R, \\
p(v)=\frac{a}{v^{\gamma}} .
\end{array}\right.
$$

We consider the Cauchy problem to (2.1) with the initial condition and far field condition

$$
\left\{\begin{array}{l}
(v, u)(0, x)=\left(v_{0}, u_{0}\right)(x), \quad x \in R \\
\lim _{x \rightarrow \pm \infty}(v, u)(t, x)=\left(v_{ \pm}, u_{ \pm}\right), \quad t \geq 0 .
\end{array}\right.
$$

Before we state the main theorem, let us recall the argument on the existence of the viscous shock wave $(V, U)(x-s t)$. Set $\xi=x-s t$. Plugging $(v, u)=(V, U)(\xi)$ into (2.1), we have the system of ordinary differential equations

$$
\left\{\begin{array}{l}
-s V_{\xi}-U_{\xi}=0 \\
-s U_{\xi}+p(V)_{\xi}=\mu_{0}\left(\frac{U_{\xi}}{V^{\alpha+1}}\right)_{\xi}
\end{array}\right.
$$

with the far field condition $(V, U)( \pm \infty)=\left(v_{ \pm}, u_{ \pm}\right)$. If we assume the existence of the solution of (2.3), then integrating the system (2.3) with respect to $\xi$, it must hold

$$
\left\{\begin{array}{l}
s V+U=s v_{ \pm}+u_{ \pm}=a_{1}: \text { constant } \\
-s U+p(V)-\mu_{0} \frac{U_{\xi}}{V^{\alpha+1}}=-s u_{ \pm}+p\left(v_{ \pm}\right)=a_{2}: \text { constant }
\end{array}\right.
$$


which in particular implies the well-known "Rankine-Hugoniot condition"

$$
\left\{\begin{array}{l}
-s\left(v_{+}-v_{-}\right)-\left(u_{+}-u_{-}\right)=0 \\
-s\left(u_{+}-u_{-}\right)+p\left(v_{+}\right)-p\left(v_{-}\right)=0 .
\end{array}\right.
$$

For any fixed $\left(v_{-}, u_{-}\right)$, regarding $v_{+}$as a prameter, there exist two families of the solution $\left(s, u_{+}\right)$of $(2.5)$ defined by

$$
s=s_{ \pm}:= \pm \sqrt{-\frac{p\left(v_{+}\right)-p\left(v_{-}\right)}{v_{+}-v_{-}}}, \quad u_{+}=u_{-}-s_{ \pm}\left(v_{+}-v_{-}\right) .
$$

In what follows, for simplicity, we focus our attention only on the case $s=s_{+}>0$ (second shock). Now plugging the first equation of (2.4) into the second one, we have the equation for $V$

$$
\left\{\begin{array}{l}
V_{\xi}=\frac{V^{\alpha+1}}{\mu_{0} s} h(V), \quad \xi \in R \\
V( \pm \infty)=v_{ \pm}
\end{array}\right.
$$

where

$$
h(V)=s^{2}\left(v_{-}-V\right)+p\left(v_{-}\right)-p(V) .
$$

Since $h(V)>0$ for $V$ taking the value between $v_{-}$and $v_{+}$, in order for the problem (2.7) to have the solution, it must hold for the case $s>0$ that

$$
v_{-}<v_{+}, \quad\left(\text { i.e. } u_{-}>u_{+}\right) .
$$

The assumption (2.9) is also well known as the "entropy condition". Conversely, if $\left(v_{ \pm}, u_{ \pm}\right)$and $s>0$ satisfy the Rankine-Hugoniot and entropy conditions (2.5), (2.9), it is easily shown that the solution $(V, U)$ of $(2.3)$ uniquely exists up to the shift of $\xi$, satisfying

$$
V_{\xi}(\xi)>0, \quad v_{-}<V(\xi)<v_{+}, \quad \xi \in R
$$

In fact, we may determine $V$ by the solution of (2.7) and $U$ by $U=u_{ \pm}+s\left(v_{ \pm}-V\right)$.

Now we fix a viscous shock wave $(V, U)$, and consider the Cauchy problem (2.1), (2.2) around a neighborhood of $(V, U)$. As for the initial data, we assume that

$$
\begin{gathered}
\left(v_{0}-V, u_{0}-U\right) \in H^{1} \cap L^{1}, \inf _{x \in R} v_{0}(x)>0, \\
\int\left(v_{0}-V\right)(x) d x=\int\left(u_{0}-U\right)(x) d x=0 .
\end{gathered}
$$

And, setting

$$
\phi_{0}(x)=\int_{-\infty}^{x}\left(v_{0}-V\right)(y) d y, \quad \psi_{0}(x)=\int_{-\infty}^{x}\left(u_{0}-U\right)(y) d y
$$

we further assume that

$$
\left(\phi_{0}, \psi_{0}\right) \in L^{2}
$$


Note that (2.13) is equivalent to $\left(\phi_{0}, \psi_{0}\right) \in H^{2}$ under the condition (2.11).

Then our main theorem is stated as follows.

Theorem 2.1 (Main Theorem). Suppose $\alpha \geq \frac{1}{2}(\gamma-1)$. Then there exist a positive constant $\varepsilon_{0}$ such that if the initial data satisfy (2.11), (2.13) and $\left\|\phi_{0}, \psi_{0}\right\|_{2} \leq \varepsilon_{0}$, the Cauchy problem (2.1), (2.2) has a unique global solution in time $(v, u)$, satisfying

$(v-V, u-U) \in C^{0}\left([0,+\infty) ; H^{1}\right), v-V \in L^{2}\left([0,+\infty) ; H^{1}\right), u-U \in L^{2}\left([0,+\infty) ; H^{2}\right)$,

and

$$
\sup _{x \in R}|(v, u)(x, t)-(V, U)(x-s t)| \rightarrow 0 \quad(t \rightarrow \infty) .
$$

3. Reformulation of the problem. First, changing the variables $(x, t) \rightarrow(\xi=$ $x-s t, t)$ in (2.1), we have

$$
\left\{\begin{array}{l}
v_{t}-s v_{\xi}-u_{\xi}=0 \\
u_{t}-s u_{\xi}+p(v)_{\xi}=\mu_{0}\left(\frac{u_{\xi}}{v^{\alpha+1}}\right)_{\xi}
\end{array}\right.
$$

Next, define the new unknown functions $(\phi, \psi)(t, \xi)$ by

$$
\phi(t, \xi)=\int_{-\infty}^{\xi}(v(t, \eta)-V(\eta)) d \eta, \quad \psi(t, \xi)=\int_{-\infty}^{\xi}(u(t, \eta)-U(\eta)) d \eta,
$$

that is,

$$
v=V+\phi_{\xi}, \quad u=U+\psi_{\xi} .
$$

Plugging the relation (3.3) into (3.1) and integrating it with respect to $\xi$ with the aid of the equation (2.3) for $(V, U)$, we have the following system in terms of $(\phi, \psi)$ :

$$
\left\{\begin{array}{l}
\phi_{t}-s \phi_{\xi}-\psi_{\xi}=0, \\
\psi_{t}-s \psi_{\xi}+p\left(V+\phi_{\xi}\right)-p(V)=\mu_{0}\left(\frac{\left(U+\psi_{\xi}\right)_{\xi}}{\left(V+\phi_{\xi}\right)^{\alpha+1}}-\frac{U_{\xi}}{V^{\alpha+1}}\right) .
\end{array}\right.
$$

Then, we consider the Cauchy problem to (3.4) with the initial data

$$
(\phi, \psi)(0)=\left(\phi_{0}, \psi_{0}\right) \in H^{2},
$$

where $\left(\phi_{0}, \psi_{0}\right)$ is defined by $(2.12)$, and we look for the small global solution in time $(\phi, \psi)$. To do that, for any interval $I$ in $R$, we define the solution space $X(I)$ by

$$
\begin{gathered}
X(I)=\left\{(\phi, \psi) \in C\left(I ; H^{2}\right) \mid \phi_{x} \in L^{2}\left(I ; H^{1}\right), \psi_{x} \in L^{2}\left(I ; H^{2}\right),\right. \\
\left.\sup _{t \in I}\|(\phi, \psi)(t)\|_{2} \leq \frac{1}{2} v_{-}\right\} .
\end{gathered}
$$

By the previous arguments as in [8], the small global solution in $X([0, \infty))$ is constructed by the combination of the local existence and the a priori estimate. Since the local solution is well understood in the previous works, let us state only the $a$ priori estimate we need.

Proposition 3.1 (a priori estimate). Suppose $\alpha \geq \frac{1}{2}(\gamma-1)$. Then there exist positive constants $\varepsilon_{0}>0$ and $C>0$ such that if $(\phi, \psi) \in X([0, T])$ is the solution of 
the Cauchy problem (3.4), (3.5) for some $T>0$ and $\sup _{t \in[0, T]}\|(\phi, \psi)(t)\|_{2} \leq \varepsilon_{0}$, it holds that for $t \in[0, T]$

$$
\|(\phi, \psi)(t)\|_{2}^{2}+\int_{0}^{t}\left(\left\|\phi_{\xi}(\tau)\right\|_{1}^{2}+\left\|\psi_{\xi}(\tau)\right\|_{2}^{2}\right) d \tau \leq C\left\|\phi_{0}, \psi_{0}\right\|_{2}^{2} .
$$

Once the Proposition 3.1 is obtained, we can show the following global existence theorem which immediately implies the main Theorem 2.1 by defining $v=V+\phi_{\xi}, u=$ $U+\psi_{\xi}$. Since the detailed argument is same as in [8], we omit it.

TheOREM 3.2. Suppose $\alpha \geq \frac{1}{2}(\gamma-1)$. Then there exist a positive constant $\varepsilon_{0}$ such that if $\left(\phi_{0}, \psi_{0}\right) \in H^{2}$ and $\left\|\phi_{0}, \psi_{0}\right\|_{2} \leq \varepsilon_{0}$, the Cauchy problem (3.4), (3.5) has a unique global solution in time $(\phi, \psi) \in \mathbf{X}([0, \infty))$ satisfying

$$
\sup _{\xi \in R}\left|(\phi, \psi)_{\xi}(t, \xi)\right| \rightarrow 0 \quad(t \rightarrow \infty)
$$

4. A priori estimate. In this section, we establish the a priori estimate Proposition 3.2. So, throughout of this section, we assume that $(\phi, \psi) \in X([0, T])$ is the solution of the Cauchy problem (3.4), (3.5) for some $T>0$ and $\alpha \geq \frac{1}{2}(\gamma-1)$. First, we rewrite the system (3.4) so that all the linearized terms at $(\phi, \psi)=(0,0)$ are collected on the left hand side and nonlinear terms on the right as in the form

$$
\left\{\begin{array}{l}
\phi_{t}-s \phi_{\xi}-\psi_{\xi}=0 \\
\psi_{t}-s \psi_{\xi}-K(V) \phi_{\xi}-\frac{\mu_{0}}{V^{\alpha+1}} \psi_{\xi \xi}=G
\end{array}\right.
$$

where

$$
K(V)=-p^{\prime}(V)+(\alpha+1) \frac{h(V)}{V}
$$

and

$$
\begin{aligned}
G= & G(\phi, \psi, V) \\
= & -\left\{p\left(V+\phi_{\xi}\right)-p(V)-p^{\prime}(V) \phi_{\xi}\right\}+\mu_{0}\left\{\frac{1}{\left(V+\phi_{\xi}\right)^{\alpha+1}}-\frac{1}{V^{\alpha+1}}\right\} \psi_{\xi \xi} \\
& +V^{\alpha+1} h(V)\left\{-\frac{1}{\left(V+\phi_{\xi}\right)^{\alpha+1}}+\frac{1}{V^{\alpha+1}}-\frac{\alpha+1}{V^{\alpha+2}} \phi_{\xi}\right\} .
\end{aligned}
$$

Here we recall

$$
s>0, \quad v_{-}<V<v_{+}, \quad V_{\xi}=\frac{V^{\alpha+1}}{\mu_{0} s} h(V)>0, \quad p^{\prime}(V)=-\gamma \frac{p(V)}{V},
$$

and note that (4.4) easily implies

$$
K(V) \geq \gamma \frac{p\left(v_{+}\right)}{v_{+}}
$$

Now it is ready to show the basic energy estimate which plays an essential role in this paper. 
Lemma 4.1. There exists a positive constant $C$ such that it holds that for $t \in[0, T]$

$$
\begin{aligned}
\|(\phi, \psi)(t)\|^{2}+\int_{0}^{t}\left(\left\|\psi_{\xi}(\tau)\right\|^{2}\right. & \left.+\left\|\sqrt{V_{\xi}} \psi(\tau)\right\|^{2}\right) d \tau \\
& \leq C\left\{\left\|\phi_{0}, \psi_{0}\right\|^{2}+\int_{0}^{t} \int|\psi \| G| d \xi d \tau\right\} .
\end{aligned}
$$

Proof. Following the idea in [3] and [9], we introduce the positive functions $\chi_{1}=\chi_{1}(V)$ and $\chi_{2}=\chi_{2}(V)$ (transform functions) which are properly determined later, and transform the variable $(\phi, \psi)$ to $(\tilde{\phi}, \tilde{\psi})$ by

$$
\phi=\chi_{1}(V) \tilde{\phi}, \quad \psi=\chi_{2}(V) \tilde{\psi} .
$$

Plugging (4.7) into (4.1), we have

$$
\left\{\begin{array}{l}
\left(\chi_{1} \tilde{\phi}\right)_{t}-s\left(\chi_{1} \tilde{\phi}\right)_{\xi}-\left(\chi_{2} \tilde{\psi}\right)_{\xi}=0 \\
\left(\chi_{2} \tilde{\psi}\right)_{t}-s\left(\chi_{2} \tilde{\psi}\right)_{\xi}-K\left(\chi_{1} \tilde{\phi}\right)_{\xi}-\frac{\mu_{0}}{V^{\alpha+1}}\left(\chi_{2} \tilde{\psi}\right)_{\xi \xi}=G .
\end{array}\right.
$$

Taking the another set of functions $W_{1}=W_{1}(V)$ and $W_{2}=W_{2}(V)$ of $V$ (weight functions) which are also determined later, we multiply the first equation of (4.8) by $W_{1} \tilde{\phi}$, the second equation by $W_{2} \tilde{\psi}$, sum them up and integrate the resultant formula with respect to $\xi$. Then we have after integration by parts,

$$
\begin{aligned}
& \frac{d}{d t} \int \frac{1}{2}\left(W_{1} \chi_{1} \tilde{\phi}^{2}+W_{2} \chi_{2} \tilde{\psi}^{2}\right) d \xi \\
& +\int \frac{s}{2}\left\{\left(W_{1}^{\prime} \chi_{1}-W_{1} \chi_{1}^{\prime}\right) \tilde{\phi}^{2}+\left(W_{2}^{\prime} \chi_{2}-W_{2} \chi_{2}^{\prime}\right) \tilde{\psi}^{2}\right\} V_{\xi} d \xi \\
& +\int\left\{\left(W_{1}^{\prime} \chi_{2}-K W_{2} \chi_{1}^{\prime}\right) V_{\xi} \tilde{\phi} \tilde{\psi}+\left(W_{1} \chi_{2}-K W_{2} \chi_{1}\right) \tilde{\phi}_{\xi} \tilde{\psi}\right\} d \xi \\
& +\int \mu_{0}\left(\frac{W_{2}}{V^{\alpha+1}} \tilde{\psi}\right)_{\xi}\left(\chi_{2} \tilde{\psi}\right)_{\xi} d \xi=\int W_{2} \tilde{\psi} G d \xi .
\end{aligned}
$$

In order for the coefficients of the cross terms $\tilde{\phi} \tilde{\psi}$ and $\tilde{\phi}_{\xi} \tilde{\psi}$ in (4.9) to vanish, we impose that

$$
W_{1}^{\prime} \chi_{2}-K W_{2} \chi_{1}^{\prime}=0, \quad W_{1} \chi_{2}-K W_{2} \chi_{1}=0 .
$$

Noting that (4.10) implies

$$
\frac{W_{1}^{\prime}}{W_{1}}=\frac{\chi_{1}^{\prime}}{\chi_{1}}
$$

we choose $\chi_{1}, \chi_{2}, W_{1}$ and $W_{2}$ for any positive function $W(V)$ of $V$ as

$$
\chi_{1}(V)=W_{1}(V)=1, \quad \chi_{2}(V)=K(V) W(V), \quad W_{2}(V)=W(V) .
$$

Plugging (4.11) into (4.9), we have

$$
\begin{gathered}
\frac{d}{d t} \int \frac{1}{2}\left(\tilde{\phi}^{2}+K W^{2} \tilde{\psi}^{2}\right) d \xi+\int\left(-\frac{s}{2} K^{\prime} W^{2} V_{\xi} \tilde{\psi}^{2}+\mu_{0}\left\{\left(\frac{W}{V^{\alpha+1}}\right)^{\prime}(K W)^{\prime} V_{\xi}^{2} \tilde{\psi}^{2}\right.\right. \\
\left.\left.+\left(\frac{K W^{2}}{V^{\alpha+1}}\right)^{\prime} V_{\xi} \tilde{\psi} \tilde{\psi}_{\xi}+\frac{K W^{2}}{V^{\alpha+1}}\left(\tilde{\psi}_{\xi}\right)^{2}\right\}\right) d \xi=\int W \tilde{\psi} G d \xi
\end{gathered}
$$


Noting $V_{\xi}=V^{\alpha+1} h(V) /\left(\mu_{0} s\right)$ and integrating the term concerning $\tilde{\psi} \tilde{\psi}_{\xi}$ by parts, (4.12) deduces

(4.13) $\frac{d}{d t} \int \frac{1}{2}\left(\tilde{\phi}^{2}+K W^{2} \tilde{\psi}^{2}\right) d \xi+\int\left(A(V) V_{\xi} \tilde{\psi}^{2}+\mu_{0} \frac{K W^{2}}{V^{\alpha+1}}\left(\tilde{\psi}_{\xi}\right)^{2}\right) d \xi=\int W \tilde{\psi} G d \xi$,

where

$$
A(V)=-\frac{s}{2} K^{\prime} W^{2}+\frac{1}{s}\left(\frac{W}{V^{\alpha+1}}\right)^{\prime}(K W)^{\prime} V^{\alpha+1} h-\frac{1}{2 s}\left(\left(\frac{K W^{2}}{V^{\alpha+1}}\right)^{\prime} V^{\alpha+1} h\right)^{\prime} .
$$

In what follows, we show the uniform positiveness of $A(V)$ for $V \in\left[v_{-}, v_{+}\right]$. To do that, using

$$
p^{\prime}(V)=-\gamma \frac{p(V)}{V}, \quad h^{\prime}(V)=-s^{2}+\gamma \frac{p(V)}{V},
$$

we represent $K, K^{\prime}$ and $K^{\prime \prime}$ in terms of $h$ and $p$ as

$$
\begin{aligned}
K(V) & =\gamma \frac{p(V)}{V}+(\alpha+1) \frac{h(V)}{V} \\
K^{\prime}(V) & =-\gamma(\gamma-\alpha) \frac{p(V)}{V^{2}}-(\alpha+1) \frac{s^{2}}{V}-(\alpha+1) \frac{h(V)}{V^{2}} \\
K^{\prime \prime}(V) & =\gamma((\gamma-\alpha)(\gamma+2)-(\alpha+1)) \frac{p(V)}{V^{3}}+2(\alpha+1) \frac{s^{2}}{V^{2}}+2(\alpha+1) \frac{h(V)}{V^{3}}
\end{aligned}
$$

Using the relations (4.15)-(4.18), we reformulate $A(V)$ as a quadratic form of $h$ as in the form

$$
A(V)=\frac{W}{2 s}\left(A_{0}(V)+A_{1}(V) h(V)+A_{2}(V) h^{2}(V)\right)
$$

where

$$
\begin{aligned}
A_{0}(V)= & \frac{\gamma^{2}(\gamma+1) p^{2}}{V^{3}} W-2\left(\frac{\gamma^{2} p^{2}}{V^{2}}-\gamma s^{2} \frac{p}{V}\right) W^{\prime}, \\
A_{1}(V)= & \left(\gamma(\gamma+2)(2 \alpha+1-\gamma) \frac{p}{V^{3}}-2 s^{2} \frac{(\alpha+1)}{V^{2}}\right) W \\
& -2\left(\gamma(2 \alpha+1-\gamma) \frac{p}{V^{2}}-2 s^{2} \frac{(\alpha+1)}{V}\right) W^{\prime}-2 \gamma \frac{p}{V} W^{\prime \prime}, \\
A_{2}(V)= & 2(\alpha+1)\left(-\frac{W}{V^{3}}+\frac{W^{\prime}}{V^{2}}-\frac{W^{\prime \prime}}{V}\right) .
\end{aligned}
$$

In order for $A_{2}(V)$ to be non-negative, we choose

$$
W(V)=V,
$$

which implies $A_{2}(V)=0$. Plugging (4.23) into (4.20) and (4.21), we obtain

$$
\begin{aligned}
A(V)= & \frac{1}{2 s}\left\{2 s^{2} \gamma p(V)+\gamma^{2}(\gamma-1) \frac{p^{2}(V)}{V}\right. \\
& \left.+2 \gamma^{2}\left(\alpha-\frac{1}{2}(\gamma-1)\right) \frac{p(V)}{V} h(V)+2(\alpha+1) s^{2} h(V)\right\}
\end{aligned}
$$


Hence, due to the assumption $\alpha \geq \frac{1}{2}(\gamma-1)$, it follows from(4.24) that

$$
A(V) \geq \operatorname{s\gamma p}\left(v_{+}\right), \quad V \in\left[v_{-}, v_{+}\right] .
$$

Thus, integrating (4.13) with respect to $t$, we can obtain

$$
\begin{aligned}
& \|(\tilde{\phi}, \tilde{\psi})(t)\|^{2}+\int_{0}^{t}\left(\left\|\tilde{\psi}_{\xi}(\tau)\right\|^{2}+\left\|\sqrt{V_{\xi}} \tilde{\psi}(\tau)\right\|^{2}\right) d \tau \\
& \leq C\left\{\left\|\tilde{\phi}_{0}, \tilde{\psi}_{0}\right\|^{2}+\int_{0}^{t} \int|\tilde{\psi} \| G| d \xi d \tau\right\} .
\end{aligned}
$$

Recalling the transformations $\phi=\tilde{\phi}, \psi=V K(V) \tilde{\psi}$, we finally have the desired inequality (4.6) from (4.26). Thus, the proof of the Lemma 4.1 is completed.

As for the estimates of the derivatives of $(\phi, \psi)$, the arguments are almost same as in [8]. So, in what follows, we show only the outline and omit the detailed arguments. We start with the $L^{2}$-estimate of $\phi_{\xi}$.

LEMma 4.2. It holds that for $t \in[0, T]$

$$
\begin{aligned}
& \left\|\phi_{\xi}(t)\right\|^{2}+\int_{0}^{t}\left\|\phi_{\xi}(\tau)\right\|^{2} d \tau \\
& \leq C\left\{\left\|\left(\phi_{0}, \psi_{0}\right)\right\|^{2}+\left\|\phi_{0, \xi}\right\|^{2}+\int_{0}^{t}\left(\int\left|\psi\|G \mid d \xi+\| G \|^{2}\right) d \tau\right\} .\right.
\end{aligned}
$$

Proof. Denote the left hand side of the first equation of (4.1) by $L_{1}(\phi, \psi)$, and the second equation by $L_{2}(\phi, \psi)$. Then, we estimate

$$
\int\left(\phi_{\xi} \partial_{\xi} L_{1}(\phi, \psi)-\frac{V^{\alpha+1}}{\mu_{0}} \phi_{\xi} L_{2}(\phi, \psi)\right) d \xi=\int-\frac{V^{\alpha+1}}{\mu_{0}} \phi_{\xi} G d \xi,
$$

which deduces

$$
\begin{aligned}
& \frac{d}{d t} \int\left(\frac{1}{2} \phi_{\xi}^{2}-\frac{V^{\alpha+1}}{\mu_{0}} \phi_{\xi} \psi\right) d \xi+\int \frac{V^{\alpha+1}}{\mu_{0}} K \phi_{\xi}^{2} d \xi \\
& =\int\left(-\frac{V^{\alpha+1}}{\mu_{0}} \phi_{\xi} G+\frac{(\alpha+1) V^{\alpha}}{\mu_{0}} s V_{\xi} \phi_{\xi} \psi+\frac{V^{\alpha+1}}{\mu_{0}} \psi_{\xi}^{2}+\frac{(\alpha+1) V^{\alpha}}{\mu_{0}} V_{\xi} \psi \psi_{\xi}\right) d \xi
\end{aligned}
$$

Using the Young's inequality and integrating (4.29) with respect to $t$, we have from $(4.29)$

$$
\begin{aligned}
\left\|\phi_{\xi}(t)\right\|^{2}+\int_{0}^{t}\left\|\phi_{\xi}(\tau)\right\|^{2} d \tau & \leq C\left\{\left\|\phi_{0, \xi}\right\|^{2}+\left\|\psi_{0}\right\|^{2}+\|\psi(t)\|^{2}\right. \\
& \left.+\int_{0}^{t}\left(\|G(\tau)\|^{2}+\left\|\sqrt{V_{\xi}} \psi(\tau)\right\|^{2}+\left\|\psi_{\xi}(\tau)\right\|^{2}\right) d \tau\right\} .
\end{aligned}
$$

Hence, combining the Lemma 4.1 with (4.30), we obtain the desired estimate (4.27). Thus the proof of the Lemma 4.2 is completed. 
LEMMA 4.3. It holds that for $t \in[0, T]$

$$
\begin{aligned}
& \left\|\psi_{\xi}(t)\right\|^{2}+\int_{0}^{t}\left\|\psi_{\xi \xi}(\tau)\right\|^{2} d \tau \\
& \leq C\left\{\left\|\psi_{0}\right\|_{1}^{2}+\int_{0}^{t}\left(\|G(\tau)\|^{2}+\left\|\phi_{\xi}(\tau)\right\|^{2}+\left\|\psi_{\xi}(\tau)\right\|^{2}\right) d \tau\right\} .
\end{aligned}
$$

Proof. We compute

$$
\int-\psi_{\xi \xi} L_{2}(\phi, \psi) d \xi=\int-\psi_{\xi \xi} G d \xi
$$

from which it easily follows that

$$
\frac{d}{d t} \int \frac{1}{2}\left(\psi_{\xi}\right)^{2} d \xi+\int\left(K \phi_{\xi} \psi_{\xi \xi}+\frac{\mu_{0}}{V^{\alpha+1}} \psi_{\xi \xi}^{2}\right) d \xi=\int-\psi_{\xi \xi} G d \xi
$$

Using the Young's inequality and integrating (4.33) with respect to $t$, we obtain

$$
\begin{aligned}
& \left\|\psi_{\xi}(t)\right\|^{2}+\int_{0}^{t}\left\|\psi_{\xi \xi}(\tau)\right\|^{2} d \tau \\
& \leq C\left\{\left\|\psi_{0, \xi}\right\|^{2}+\int_{0}^{t}\left(\|G(\tau)\|^{2}+\left\|\phi_{\xi}(\tau)\right\|^{2}+\left\|\psi_{\xi}(\tau)\right\|^{2}\right) d \tau\right\} .
\end{aligned}
$$

Due to the Lemma 4.2, the desired estimate (4.31) easily follows. Thus the proof of the Lemma 4.3 is completed.

Similarly, calculating the formulas corresponding to (4.28) and (4.32), that is,

$$
\int_{0}^{t} \int\left(\phi_{\xi \xi} \partial_{\xi}^{2} L_{1}(\phi, \psi)-\frac{V^{\alpha+1}}{\mu_{0}} \phi_{\xi \xi} L_{2}(\phi, \psi)\right) d \xi d \tau=\int_{0}^{t} \int-\frac{V^{\alpha+1}}{\mu_{0}} \phi_{\xi \xi} G_{\xi} d \xi d \tau
$$

and

$$
\int_{0}^{t} \int-\psi_{\xi \xi \xi} \partial_{\xi} L_{2}(\phi, \psi) d \xi d \tau=\int_{0}^{t} \int-\psi_{\xi \xi \xi} G_{\xi} d \xi d \tau,
$$

we can show the following lemmas concerning with the $L^{2}$-estimates of $\left(\phi_{\xi \xi}, \psi_{\xi \xi}\right)$.

LEMMA 4.4. It holds that for $t \in[0, T]$

$$
\begin{aligned}
\left\|\phi_{\xi \xi}(t)\right\|^{2}+ & \int_{0}^{t}\left\|\phi_{\xi \xi}(\tau)\right\|^{2} d \tau \\
& \leq C\left\{\left\|\left(\phi_{0}, \psi_{0}\right)\right\|_{1}^{2}+\left\|\phi_{0, \xi \xi}\right\|^{2}+\int_{0}^{t}\left(\int\left|\psi\|G \mid d \xi+\| G \|_{1}^{2}\right) d \tau\right\} .\right.
\end{aligned}
$$

LEMMA 4.5. It holds that for $t \in[0, T]$

$$
\begin{aligned}
\left\|\psi_{\xi \xi}(t)\right\|^{2}+ & \int_{0}^{t}\left\|\psi_{\xi \xi \xi}(\tau)\right\|^{2} d \tau \\
& \leq C\left\{\left\|\left(\phi_{0}, \psi_{0}\right)\right\|_{2}^{2}+\int_{0}^{t}\left(\int\left|\psi\|G \mid d \xi+\| G \|_{1}^{2}\right) d \tau\right\} .\right.
\end{aligned}
$$


It follows from the Lemma 4.1-Lemma 4.5 that

$$
\begin{aligned}
\|(\phi, \psi)(t)\|_{2}^{2} & +\int_{0}^{t}\left(\left\|\phi_{\xi}(\tau)\right\|_{1}^{2}+\left\|\psi_{\xi}(\tau)\right\|_{2}^{2}\right) d \tau \\
& \leq C\left\{\left\|\left(\phi_{0}, \psi_{0}\right)\right\|_{2}^{2}+\int_{0}^{t} \int\left|\psi\left\|G \mid d \xi d \tau+\int_{0}^{t}\right\| G \|_{1}^{2} d \tau\right\}\right.
\end{aligned}
$$

Finally, as for the estimate for the nonliner terms $G$, noting that

$$
\frac{1}{V+\phi_{\xi}} \leq \frac{2}{v_{-}}, \quad(\phi, \psi) \in X([0, T])
$$

and using the Sovolev's inequality, it is very standard to have the following lemma.

LEMma 4.6. For any $\varepsilon_{0} \in\left(0, \frac{1}{2} v_{-}\right]$, if $\sup _{t \in[0, T]}\|(\phi, \psi)(t)\|_{2} \leq \varepsilon_{0}$, then it holds that for $t \in[0, T]$

$$
\int_{0}^{t} \int\left|\psi\left\|G \mid d \xi d \tau+\int_{0}^{t}\right\| G \|_{1}^{2} d \tau \leq C \varepsilon_{0} \int_{0}^{t}\left(\left\|\phi_{\xi}(\tau)\right\|_{1}^{2}+\left\|\psi_{\xi}(\tau)\right\|_{2}^{2}\right) d \tau .\right.
$$

Then, from (4.39) and (4.40), we eventually have

$$
\|(\phi, \psi)(t)\|_{2}^{2}+\left(1-C \varepsilon_{0}\right) \int_{0}^{t}\left(\left\|\phi_{\xi}(\tau)\right\|_{1}^{2}+\left\|\psi_{\xi}(\tau)\right\|_{2}^{2}\right) d \tau \leq C\left\|\left(\phi_{0}, \psi_{0}\right)\right\|_{2}^{2}
$$

Thus, choosing $\varepsilon_{0}$ is properly small in (4.41), the proof of the Proposition 3.2, that is the desired a priori estimate, is completed.

5. A remark. In this section, we give a remark that our arguments in the previous sections can be extended to the models with more general viscosity coefficient. In the book of Chapman-Cowling [2], the viscosity coefficient is given in more general form as

$$
\mu=\bar{\mu} \theta^{\frac{1}{2}} F(\theta)
$$

It is noted that $F(\theta)=1$ for the Hard sphere Model, and $F(\theta)=\theta^{\frac{2}{(s-1)}}$ for the Cutoff inverse power force Model in (3.2). As a typical model whose $F$ is not a power function of $\theta$, the Sutherland's model is well known where

$$
F(\theta)=\frac{1}{1+\frac{s_{0}}{\theta}}, \quad\left(s_{0}>0: \text { Sutherland's constant }\right) .
$$

Even for the general case (5.1), if we also take

$$
\chi_{1}(V)=W_{1}(V)=1, \quad \chi_{2}(V)=K(V) W(V), \quad W_{2}(V)=W(V)=V
$$


as in the proof of the Lemma 4.1, then after long calculations we can have the following which corresponds to (4.24):

$$
\begin{aligned}
A(V)= & \frac{1}{2 s}\left\{2 s^{2} \gamma p(V)+\gamma^{2}(\gamma-1) \frac{p^{2}(V)}{V}\right. \\
& +\left.2 s^{2} h(V)\left(\frac{\gamma+1}{2}+(\gamma-1) \theta \frac{F^{\prime}}{F}-(\gamma-1)^{2} \theta\left(\theta \frac{F^{\prime}}{F}\right)^{\prime}\right)\right|_{\theta=(a / R) V^{1-\gamma}} \\
& +\left.2 \gamma(\gamma-1) h(V) \frac{p(V)}{V}\left(\gamma \theta \frac{F^{\prime}}{F}+(\gamma-1) \theta\left(\theta \frac{F^{\prime}}{F}\right)^{\prime}\right)\right|_{\theta=(a / R) V^{1-\gamma}} \\
& \left.-\left.(\gamma-1)^{2} \frac{h^{2}(V)}{V}\left(\gamma \theta\left(\theta \frac{F^{\prime}}{F}\right)^{\prime}+(\gamma-1) \theta^{2}\left(\theta \frac{F^{\prime}}{F}\right)^{\prime \prime}\right)\right|_{\theta=(a / R) V^{1-\gamma}}\right\}
\end{aligned}
$$

Therefore, if it holds

$$
\left\{\begin{array}{l}
\frac{\gamma+1}{2}+(\gamma-1) \theta \frac{F^{\prime}}{F}-(\gamma-1)^{2} \theta\left(\theta \frac{F^{\prime}}{F}\right)^{\prime} \geq 0 \\
\gamma \theta \frac{F^{\prime}}{F}+(\gamma-1) \theta\left(\theta \frac{F^{\prime}}{F}\right)^{\prime} \geq 0 \\
\gamma \theta\left(\theta \frac{F^{\prime}}{F}\right)^{\prime}+(\gamma-1) \theta^{2}\left(\theta \frac{F^{\prime}}{F}\right)^{\prime \prime} \leq 0
\end{array}\right.
$$

then as in the section 4 the desired a priori estimate holds, and eventually the asymptotic stability of the viscous shock follows for small initial perturbations with integral zero. Finally, we note that as for the Sutherland's model (5.2) the direct computation shows the condition (5.4) holds for $\gamma \in[1,2]$.

\section{REFERENCES}

[1] B. Barker, J. Humpherys, O. Laffite, K. Rudd and K. Zumbrun, Stability of isentropic Navier-Stokes shocks, Appl. Math. Lett., 21 (2008), pp. 742-747.

[2] S. Chapman and T. Cowling, The mathematical theory of non-uniform gases, 3rd ed. London, Cambridge University Press (1970).

[3] I. Hashimoto And A. Matsumura, Large-time behavior of solutions to an initial-boundary value problem on the half line for scalar vicous conservation law, Met. Appl. Anal., 14 (2007), pp. 45-60.

[4] J. Humpherys, O. Laffite And K. Zumbrun, Stability of isentropic viscous shock profiles in the high-Mach number limit, Comm. Math. Phys., 293 (2010), pp. 1-36.

[5] T.-P. LIU AND Y. ZENG, Time-asymptotic behavior of wave propagation around a viscous shock profile, Comm. Math. Phys., 290 (2009), pp. 23-82.

[6] S. Kawashima, A. Matsumura and T. Nishida, On the fluid-dynamical approximation to the Boltzmann equation at the level of the Navier-Stokes equation, Comm. Math. Phys., 70 (1979), pp. 97-124.

[7] C. Mascia AND K. Zumbrun, Stability of small-amplitude shock profiles of symmetric hyperbolic-parabolic systems, Comm. Pure Appl. Math., 57 (2004), pp. 841-876.

[8] A. Matsumura And K. Nishihara, On the stability of traveling wave solutions of a onedimensional model system for compressible viscous gas, Japan J. Appl. Math., 2 (1985), pp. $17-25$

[9] A. Matsumura And M. MeI, Nonlinear stability of viscous shock profile for a non-convex system of viscoelasticity, Osaka J. Math., 34 (1997), pp. 589-603. 